\title{
Review \\ Imaging in breast cancer: Magnetic resonance imaging
} Constance D Lehman ${ }^{1}$ and Mitchell D Schnall ${ }^{2}$

\author{
1'Department of Radiology, University of Washington, Seattle Cancer Care Alliance, Seattle, WA, USA \\ ${ }^{2}$ Department of Radiology, University of Pennsylvania, Philadelphia, PA, USA
}

Corresponding author: Constance D Lehman, lehman@seattlecca.org

Published: 5 August 2005

This article is online at http://breast-cancer-research.com/content/7/5/215

(c) 2005 BioMed Central Ltd

\begin{abstract}
Over the past 5 years there has been a marked increase in the use of magnetic resonance imaging (MRI) of the breast. Multiple research studies have confirmed improved cancer detection, diagnosis, and evaluation of response to therapy with breast MRI compared with mammography and ultrasound. As this exciting new technology advances, focused work in optimal scan protocols, appropriate clinical applications, and image interpretation are needed. Both the potential benefits and harms need to be evaluated to guide optimal use of this imaging modality in select patient populations.
\end{abstract}

\section{History of magnetic resonance imaging of the breast}

Some of the first images of the body produced with magnetic resonance imaging (MRI) were of the breast [1]. However, by the mid-1980s most investigators had concluded that there was little clinical utility for MRI in detecting or diagnosing breast cancer. The application of contrast agents to breast imaging, first published by Heywang and colleagues [2], changed that thinking and revealed that breast cancers, in comparison with normal breast tissue, were enhanced significantly with standard gadolinium contrast agents. Heywang's reports were followed closely by those of Kaiser and Zeitler [3], who also found contrast-enhanced magnetic resonance images useful in breast cancer diagnosis but by using a very different technique. Whereas Heywang and colleagues acquired one pre-contrast and two post-contrast sequences of a single breast, permitting high spatial resolution with a three-dimensional gradient echo technique, Kaiser and Zeitler obtained one pre-contrast and multiple post-contrast images of both breasts, permitting high temporal resolution.

This work by Heywang and Kaiser in the 1980s established that contrast-enhanced MRI could distinguish benign from malignant breast tissue; additional reports, including those by Harms and Kuhl in the 1990s [4,5], contributed to our
Breast Cancer Research 2005, 7:215-219 (DOI 10.1186/bcr1309)

understanding of the optimal methods of image acquisition. Two basic camps were established, one focusing on the rapid acquisition of images of both breasts after contrast injection (high temporal resolution of the 'dynamic' school) and the other focusing on three-dimensional gradient echo imaging with thin slices through one breast (high spatial resolution of the 'static' school). The dynamic school tended to use image subtraction to suppress the high signal from fat, whereas the static school tended to eliminate the fat signal by more time-consuming methods of suppression. The dynamic school, more popular in Europe, helped to develop methods to evaluate various enhancement profiles over time, and the static school, more popular in the USA, helped to develop methods to distinguish morphologic features of malignant and benign lesions [6]. By 2000, most agreed that both high temporal and high spatial resolution were important in gaining information about both the pharmacokinetics and morphology of breast lesions. Importantly, current technology permits acquisition protocols that provide both high spatial resolution ( $\leq 3 \mathrm{~mm}$ slices with $\leq 1 \mathrm{~mm}$ in-plane spatial resolution) and high temporal resolution (fat-suppressed $T_{1}$ acquisitions covering both breasts in $\leq 2 \mathrm{~min}$ ).

\section{Breast MRI acquisition techniques}

There are numerous acceptable methods of image acquisition, and no single method has proven superior to another. However, there are guidelines considered by most experts to be reasonable minimum requirements for achieving acceptable image quality.

A dedicated breast surface coil should always be used. Both unilateral and bilateral coils are available. Bilateral imaging has obvious advantages of cost, time, and patient convenience. Most coils sold today are for bilateral imaging and most are open, allowing for access to the breast tissue for MRI-guided interventions. The vast majority of published studies of breast MRI have been conducted on 1.5 T magnets, but there are some reports from $1.0 \mathrm{~T}$ scanners. 
In 2003, the 4th edition of the manual for the American College of Radiology Breast Imaging Reporting and Data System (BI$\operatorname{RADS}^{\circledR}$ ) included a section dedicated to the performance and reporting of breast MRI [7]. The committee acknowledged that no single method of image acquisition had been proven superior to others, but that reporting of breast MRI should include field strength, pre-contrast and post-contrast sequences used, method of fat suppression, and postprocessing performed (subtractions, axial, sagittal, coronal reconstructions, and/or maximum intensity projections).

\section{Breast MR image interpretation}

The American College of Radiology BI-RADS MRI lexicon for breast imaging includes detailed language for describing morphology and kinetics of lesions. All suspicious enhancing areas should be described as a focus or foci, mass or nonmass-like enhancement. Foci are typically less than $5 \mathrm{~mm}$ in diameter, whereas masses have defined convex margins. All mass descriptions should include reporting of mass shape, margin and internal enhancement. Non-mass-like enhancement descriptions should include distribution, internal enhancement and symmetry. Associated findings (such as edema, adenopathy, cysts, and skin or chest wall involvement) should be reported and kinetic curve assessment of all lesions described. Kinetic curve assessment should include initial peak enhancement (slow, medium, or rapid) and delayed-phase (persistent, plateau, or washout) analyses.

\section{Clinical applications of breast MRI}

Numerous reports evaluating the potential role of breast MRI in defined patient populations have been published. These studies cover the spectrum of cancer detection, diagnosis, and response to treatment evaluation, and include women with a mammographic or palpable abnormality, axillary adenopathy but unknown primary, current cancer diagnosis, and women at high risk for breast cancer.

\section{Further evaluation of mammographic or palpable abnormality}

Initially, clinical studies of breast MRI focused on the potential role of $\mathrm{MRI}$ in further evaluation of a mammographic or palpable lesion. It was proposed that MRI could reduce the number of unnecessary biopsies recommended from traditional work-ups of mammographic or palpable lesions. However, although MRI was shown to have very high sensitivity it was not $100 \%$ sensitive and it demonstrated only moderate specificity. In 2004 the International Breast MRI Consortium published the largest $(n=821)$ multicenter study so far of patients recommended for biopsy based on abnormal mammogram, ultrasound, or clinical breast exam [8]. This study demonstrated that although MRI had very high sensitivity, $12 \%$ of cancers identified by mammography or clinical breast exam were negative on MRI. The authors concluded that MRI should not be used to overrule a use of dynamic MRI in this patient population did not improve accuracy compared with high-spatial-resolution threedimensional MRI alone.

\section{Evaluation of extent of disease}

Although negative or benign MRI findings cannot replace a recommendation for biopsy based on traditional methods, MRI does seem to be important in the assessment of extent of disease in patients with a recent diagnosis of breast cancer. This application was pioneered by Harms and colleagues [9] and confirmed by multiple reports over the past 15 years including that by Bedrosian and colleagues [10], all demonstrating that MRI can identify otherwise occult multicentric and multifocal disease in women with breast cancer. Harms compared results from in vivo MR images with serially sectioned pathologic analyses in 30 mastectomy specimens. MRI detected additional disease in $37 \%$ of specimens. Several subsequent reports confirmed the findings of Harms and colleagues, including a report from the University of Pennsylvania [11] that MRI changed management of $23 \%$ of patients scheduled for breast conserving therapy. In the largest multisite study so far, the International Breast MRI Consortium reported on 426 women with a current cancer diagnosis. MRI identified additional disease at least $2 \mathrm{~cm}$ from the index malignant lesion in $18 \%$ of patients [12].

The advantage of MRI in determining the extent of disease has also been demonstrated in studies evaluating the contralateral breast. In a recent study of 239 women with a breast cancer diagnosis who underwent prophylactic contralateral mastectomy (no known disease in the contralateral breast), $4.6 \%$ of women had cancer identified by pathology [13]. Interestingly, seven clinical studies of women with a recent cancer diagnosis have found that, on average, $4 \%$ of women will have otherwise occult contralateral cancers identified by MRI at the time of the initial breast cancer diagnosis [14]. These data suggest that most contralateral cancers can be detected at the time of the initial breast cancer diagnosis (Fig. 1).

\section{Malignant adenopathy, unknown primary}

A small percentage (1 to $2 \%$ ) of breast cancer patients present with axillary adenopathy, unknown primary. Current treatment recommendations for these patients is mastectomy. However, MRI will detect the occult cancer in 75 to $85 \%$ of patients, allowing many of these to have lumpectomy rather than mastectomy [15].

\section{Evaluation of response to neoadjuvant chemotherapy}

In the mid-1990s, Gilles and colleagues [16] reported that MRI was superior to mammography and clinical breast exam in evaluating response to neoadjuvant chemotherapy. Subsequent studies supported these findings but cautioned that the false negative rate of $M R I$ is increased after chemotherapy and MRI cannot exclude microscopic disease $[17,18]$. 

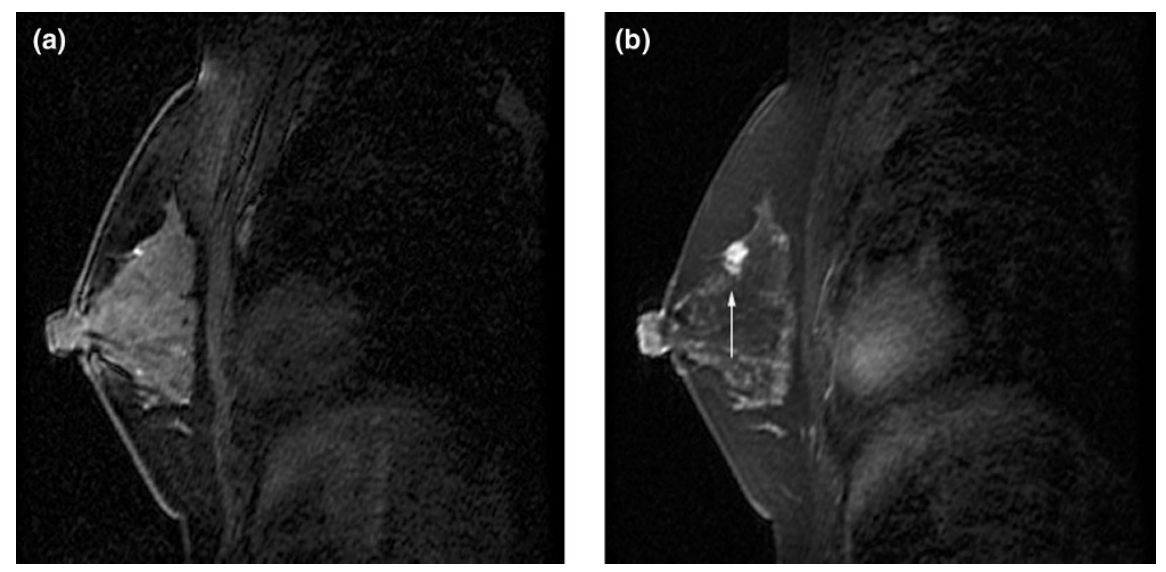

MRI results in a woman 52 years old with recent diagnosis of right breast cancer. Left mammogram negative. Pre-contrast (a) and post-contrast (b) enhanced sagittal MR images reveal an $8 \mathrm{~mm}$ enhancing mass at 12 o'clock in the left breast (arrowed). Core needle biopsy confirmed infiltrating ductal carcinoma. Final pathology from lumpectomy demonstrated an $8 \mathrm{~mm}$ infiltrating ductal carcinoma; sentinel lymph node negative.

\section{Figure 2}
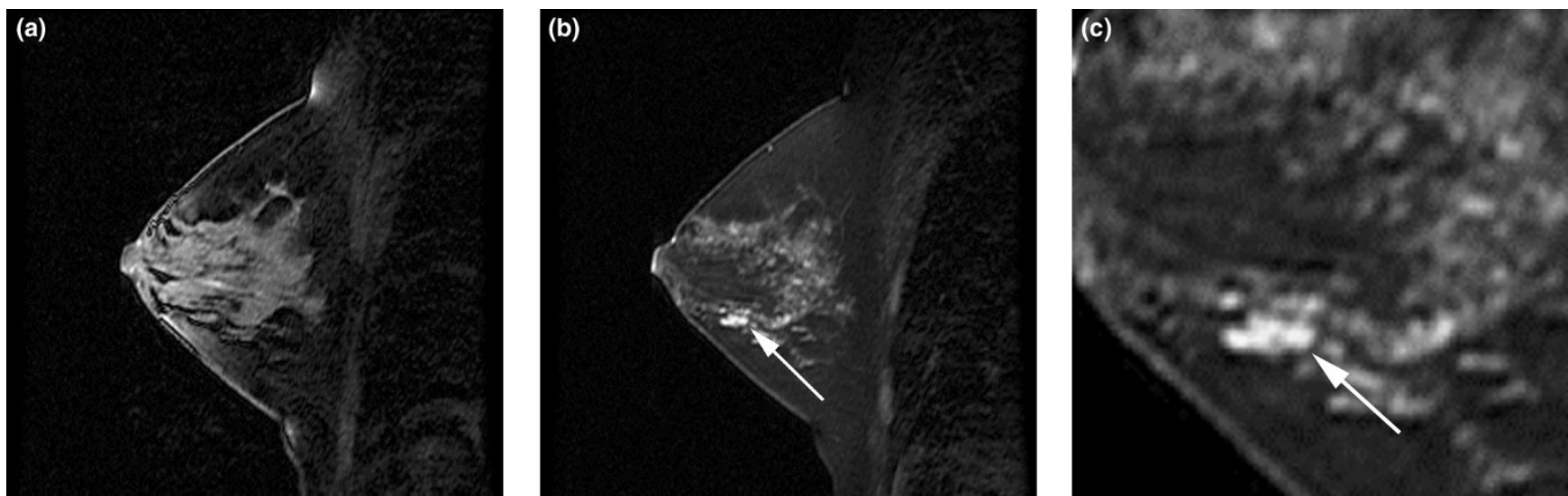

MRI results in a 46 year old woman at high risk for breast cancer. Sagittal pre-contrast $T_{2}$ (a), post-contrast $T_{1}$ (b) and magnified view (c) of $8 \times 3 \times 3$ linear focus of enhancement in left breast at six o'clock (arrowed). The lesion was negative on mammography and screening ultrasound. Pathology proved infiltrating ductal carcinoma.

\section{Screening women at high risk for breast cancer}

Although all women are at risk for developing breast cancer, there are subgroups of women who can be identified by genetic testing or by risk modeling who are at significantly increased risk for breast cancer. For example, women who are carriers of the BRCA1 mutation have an approximately $85 \%$ lifetime risk of developing breast cancer. In addition, women at increased risk tend to develop breast cancer at a younger age when mammography is less sensitive, probably because of increased mammographic density and increased growth rates of tumors in women of younger age (Fig. 2).

Multiple studies published since 2000 demonstrate that screening MRI can detect otherwise occult breast cancers in women at high risk (Table 1) [19-25]. The first study published on screening MRI in high-risk women was by Kuhl and colleagues [19], who screened 192 women with mammography, MRI, and ultrasound. In that study, MRI detected 6 cancers in 192 women (3\%) that were occult on both mammography and ultrasound. The largest screening MRI study so far [24] reported on 1,909 women at increased risk in the Netherlands, with 51 women diagnosed with cancer. The sensitivities of clinical breast examination, mammography, and MRI were $17.9 \%, 33 \%$, and $79.5 \%$, respectively. The overall discriminating capacity of MRI was significantly better than mammography as assessed by receiver operator curves (area under the curve: 0.83 for MRI versus 0.69 for mammography). 


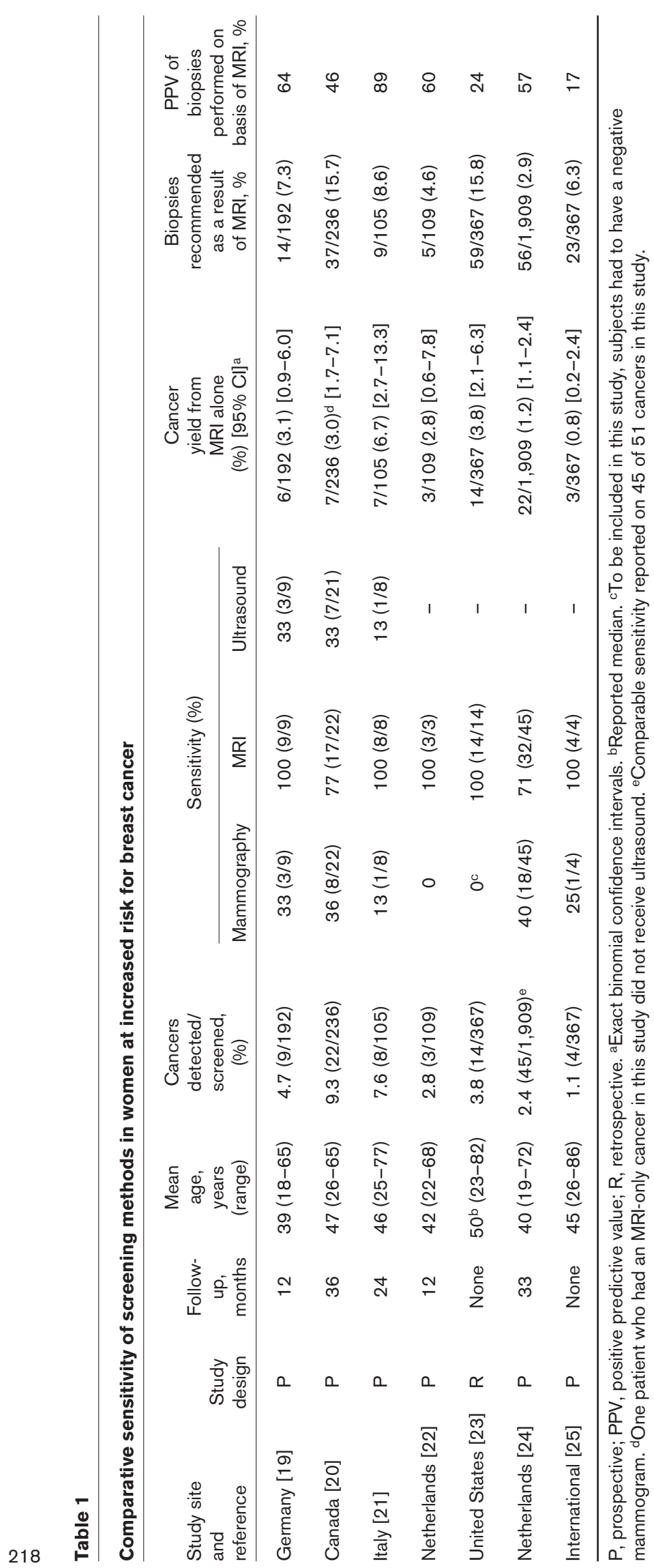

Although in all studies so far MRI sensitivity has been uniformly high, the specificity and positive predictive value of biopsies vary widely. The rates of biopsies performed in women undergoing screening MRI have ranged from $2.9 \%$ to $16 \%$, with positive predictive value of those biopsies ranging from $17 \%$ to $89 \%$. It is interesting that MRI specificity seems to improve after the first round of screening. Warner and colleagues [20] reported recall rates decreasing during the first, second and third rounds of MRI screening from $17 \%$ to $10 \%$ to $7 \%$.

MRI has not been studied in the general population as a screening tool, and the results from MRI screening of highrisk women may not apply to women at average risk. The high cost of MRI (about 10 times the cost of mammography) and its variable specificity currently prohibit its routine use for screening general populations.

Two specific populations of women are considered to have relative contraindications to contrast-enhanced MRI: pregnant and nursing women. Other than early animal studies showing adverse effects on embryo development, there is little information about the risks of contrast-enhanced imaging during pregnancy. Little is also known about the transfer and effects of this agent on nursing infants. Makers of the agent gadodiamide (Omniscan; Amersham Health) identify it as a Category $\mathrm{C}$ drug and recommend caution in its use in these two specific populations, noting that contrast-enhanced imaging should be conducted on pregnant women only when the benefits of the exam are considered to outweigh the risks to the fetus. For nursing mothers, the current recommendation is to wait 24 hours after injection before resuming nursing.

\section{Conclusion}

The use of breast MRI is increasing rapidly as this exciting technology improves and as data continue to become available supporting the value of this tool in select patient populations. Breast MRI is highly sensitive, with an acceptable specificity compared with other imaging modalities. Although MRI clearly detects cancers occult to mammography, ultrasound, and clinical breast exam, the impact of MRI on breast cancer recurrence or mortality has not been studied. Analyses of cost-effectiveness of MRI in distinct patient populations need to be performed. There is significant work to be done to optimize the application and performance of breast MRI. Research to clarify optimal acquisition protocols is needed. Recent work in breast MRI in 3 T magnets is very exciting and holds promise for even higher spatial and temporal resolution by providing a better signal : noise ratio. MRI spectroscopy, reviewed in another article in this series [26], may improve the specificity of MRI and might possibly predict the response to therapy and/or evaluate the very early response to chemotherapy. Novel contrast agents are being developed that may provide more sensitive and more specific discrimination of benign from malignant lesions. These rapidly advancing areas of research 


\section{This article is part of a review series on Imaging in breast cancer, edited by David A Mankoff.}

Other articles in the series can be found online at http://breast-cancer-research.com/articles/ review-series.asp?series=BCR_Imaging

hold great promise for continued improvements in the earlier and more accurate diagnosis of breast cancer.

\section{Competing interests}

$\mathrm{CL}$ has a consultant agreement with General Electric Company, for which her duties include giving lectures and developing teaching programs in breast imaging. MS serves as a consultant to Ethicon and to MedRad. He is also the recipient of grant and research support from Siemens Medical Solutions, royalties from USA Instruments, and past honoraria from GE Healthcare for work related to breast imaging.

\section{Acknowledgements}

We thank Sue Peacock and Tamara Fernando for their assistance in manuscript preparation.

\section{References}

1. Mansfield P, Morris P, Ordidge R, Coupland R, Bishop H, Blamey $\mathrm{R}$ : Carcinoma of the breast imaged by nuclear magnetic resonance (NMR). Br J Radiol 1979, 52:242-243.

2. Heywang S, Fenzl G, Hahn D, Krischke I, Edmaier M, Eiermann W, Bassermann R: MR imaging of the breast: comparison with mammography and ultrasound. J Comput Assist Tomogr 1986, 10:615-620.

3. Kaiser W, Zeitler E: MR imaging of the breast: fast imaging sequences with and without Gd-DTPA. Preliminary observations. Radiology 1989, 170:681-686.

4. Harms SE, Flamig DP: MR imaging of the breast: technical approach and clinical experience. Radiographics 1993, 13:905912

5. Kuhl CK, Mielcareck P, Klaschik S, Leutner C, Wardelmann E, Gieseke J, Schild HH: Dynamic breast MR imaging: are signal intensity time course data useful for differential diagnosis of enhancing lesions? Radiology 1999, 211:101-110.

6. Kuhl CK, Schild HH: Dynamic image interpretation of MRI of the breast. J Magn Reson Imaging 2000, 12:965-974.

7. American College of Radiology: ACR BI-RADS ${ }^{\circledR}$ - MRI. ACR Breast Imaging Reporting and Data System. In Breast Imaging Atlas. Reston, VA: American College of Radiology; 2003.

8. Bluemke D, Gatsonis C, Chen M, DeAngelis G, DeBruhl N, Harms S, Heywang-Kobrunner S, Hylton N, Kuhl C, Lehman C, et al:: Magnetic resonance imaging of the breast prior to biopsy. JAMA 2004, 292:2735-2742.

9. Harms SE, Flamig DP, Hesley KL, Meiches MD, Jensen RA, Evans WP, Savino DA, Wells RV: MR imaging of the breast with rotating delivery of excitation off resonance: clinical experience with pathologic correlation. Radiology 1993, 187:493-501.

10. Bedrosian I, Mick R, Orel SG, Schnall M, Reynolds C, Spitz FR, Callans LS, Buzby GP, Rosato EF, Fraker DL, et al.: Changes in the surgical management of patients with breast carcinoma based on preoperative magnetic resonance imaging. Cancer 2003, 98:468-473.

11. Tan JE OS, Schnall MD, Schultz DJ, Solin LJ: Role of magnetic resonance imaging and magnetic resonance imaging guided surgery in the evaluation of patients with early-stage breast cancer for breast conservation treatment. $\mathrm{Am} J \mathrm{C}$ Clin Oncol 1999, 22:414-418.
12. Schnall MD, Blume J, Bluemke D, Smazal S, Deangelis G, Harms S, Kuhl C, Hylton N, Gatsonis C, International Breast MRI Consortium: MRI detection of multi focal breast carcinoma: report from the International Breast MRI Consortium. J Clin Oncol 2004, 22 (Suppl): 14 S.

13. Goldflam K, Hunt KK, Gershenwald JE, Singletary SE, Mirza N, Kuerer HM, Babiera GV, Ames FC, Ross MI, Feig BW, et al:: Contralateral prophylactic mastectomy. Predictors of significant histologic findings. Cancer 2004, 101:1977-1986.

14. Lehman CD, Blume JD, Thickman D, Bluemke DA, Pisano E, Kuhl $\mathrm{K}$, Julian $\mathrm{T}$, Hylton $\mathrm{N}$, Weatherall $\mathrm{P}$, O'Loughlin $\mathrm{M}$, et al.: The added cancer yield of MRI in screening the contralateral breast of women recently diagnosed with breast cancer: results from the International Breast Magnetic Resonance Consortium (IBMC) trial. J Surg Oncol 2005, in press.

15. Orel S, Weinstein S, Schnall M, Reynolds C, Schuchter L, Fraker $\mathrm{D}$, Solin L: Breast MR imaging in patients with axillary node metastases and unknown primary malignancy. Radiology 1999, 212:543-549.

16. Gilles R, Guinebretiere JM, Toussaint C, Spielman M, Rietjens M, Petit JY, Contesso G, Masselot J, Vanel D: Locally advanced breast cancer: contrast-enhanced subtraction MR imaging of response to preoperative chemotherapy. Radiology 1994, 191: 633-638.

17. Rosen EL, Blackwell KL, Baker JA, Soo MS, Bentley RC, Yu D, Samulski TV, Dewhirst MW: Accuracy of MRI in the detection of residual breast cancer after neoadjuvant chemotherapy. AJR Am J Roentgenol 2003, 181:1275-1282.

18. Partridge SC, Gibbs JE, Lu Y, Esserman LJ, Sudilovsky D, Hylton NM: Accuracy of MR imaging for revealing residual breast cancer in patients who have undergone neoadjuvant chemotherapy. AJR Am J Roentgenol 2002, 179:1193-1199.

19. Kuhl CK, Schmutzler RK, Leutner CC, Kempe A, Wardelmann E, Hocke A, Maringa M, Pfeifer U, Krebs D, Schild HH: Breast MR imaging screening in 192 women proved or suspected to be carriers of a breast cancer susceptibility gene: preliminary results. Radiology 2000, 215:267-279.

20. Warner E, Plewes DB, Hill KA, Causer PA, Zubovits JT, Jong RA Cutrara MR, DeBoer G, Yaffe MJ, Messner SJ, et al: Surveillance of BRCA1 and BRCA2 mutation carriers with magnetic resonance imaging, ultrasound, mammography, and clinical breast examination. JAMA 2004, 292:1317-1325.

21. Podo F, Sardanelli F, Canese R, D'Agnolo G, Natali PG, Crecco M, Grandinetti ML, Musumeci R, Trecate G, Bergonzi S, et al.: The Italian multi-centre project on evaluation of MRI and other imaging modalities in early detection of breast cancer in subjects at high genetic risk. J Exp Clin Cancer Res 2002, 21 (Suppl 3):115-124.

22. Tilanus-Linthorst MM, Obdeijn IM, Bartels $\mathrm{KC}$, de Koning $\mathrm{HJ}$, Oudkerk M: First experiences in screening women at high risk for breast cancer with MR imaging. Breast Cancer Res Treat 2000, 63:53-60.

23. Morris $E A$, Liberman $L$, Ballon $D J$, Robson $M$, Abramson $A F$ Heerdt A, Dershaw DD: MRI of occult breast carcinoma in a high-risk population. AJR Am J Roentgenol 2003, 181:619626.

24. Kriege M, Brekelmans CT, Boetes $C$, Besnard PE, Zonderland HM, Obdeijn IM, Manoliu RA, Kok T, Peterse H, Tilanus-Linthorst MM, et al.: Efficacy of MRI and mammography for breastcancer screening in women with a familial or genetic predisposition. N Engl J Med 2004, 351:427-437.

25. Lehman CD, Blume JD, Weatherall P, Thickman D, Hylton N, Warner E, Pisano E, Schnitt SJ, Gatsonis C, Schnall M: Screening women at high risk for breast cancer with mammography and magnetic resonance imaging. Cancer 2005, 103:18981905.

26. Bolan PJ, Nelson MT, Yee D, Garwood M: Imaging in breast cancer: magnetic resonance spectroscopy. Breast Cancer Res 2005, 7:149-152. 